\title{
Performance Prediction and Irreversibility Analysis of a Thermoelectric Refrigerator with Finned Heat Exchanger
}

\author{
F. Meng, L. Chen* And F. Sun \\ College of Naval Architecture and Power, Naval University of Engineering, Wuhan 430033, P.R. China
}

(Received December 18, 2010; in final form April 1, 2011)

\begin{abstract}
A developed model of commercial thermoelectric refrigerators with finned heat exchanger is established by introducing finite time thermodynamics. A significant novelty is that physical properties, dimension parameters, temperature parameters and flow parameters are all taken into account in the model. Numerical studies and comparative investigation on the performance of a typical commercial water-cooling thermoelectric refrigerator which consists of 127 thermoelectric elements, are performed for cooling load and coefficient of performance. The results show that the maximum cooling load is $2.33 \mathrm{~W}$ and the maximum coefficient of performance is 0.54 when the cooling temperature difference is $10 \mathrm{~K}$. Comparing the simulation results of several models, it is found that the heat convection of the heat exchanger and the heat leakage through the air gap are the main factors, which cause irreversibility and decrease the performance. Moreover, the performance can be improved by optimizing the length and cross-section area of thermoelectric elements. The model and calculation method may be applied to not only the analysis and performance prediction of practical thermoelectric refrigerators, but also the design and optimization of heat exchangers.
\end{abstract}

PACS: 05.70.-a, 05.60.Cd, 05.70.Ln

\section{Introduction}

Different from conventional refrigeration systems, thermoelectric refrigeration [1], based on the Peltier effect, does not require any compressor, expansion valves, absorbers, condensers or solution pumps. Moreover, it does not require working fluids or any moving parts, which is friendly to the environment and results in an increase in reliability. It simply uses electrons rather than refrigerants as a heat carrier. Nowadays, thermoelectric refrigeration devices have a distinct place in medical applications, electronic applications, scientific equipment and other applications, where a high-precision temperature-control is essential [2,3]. More widespread use of thermoelectrics requires not only improving the intrinsic energy-conversion efficiency of the materials [4], but also modeling and analyzing of the thermoelectric device systems $[5,6]$.

In general, conventional non equilibrium thermodynamics [7-9] is used to analyze the performance of single-stage one or multiple-element thermoelectric refrigerators. Considering the inner structure of a thermoelec-

\footnotetext{
* corresponding author; e-mail:

lgchenna@yahoo.com, lingenchen@hotmail.com
}

tric refrigerator, a significant increase in the cooling load or coefficient of performance (COP) can be achieved by modifying the dimensions of the thermoelectric elements [10]. Yamanashi [11], Chen et al. [12], Huang et al. [13], and Xuan [14] analyzed the thermoelectric refrigerators in various ways using non equilibrium thermodynamics.

Thermoelectric devices cannot be used independently. They should be connected with heat exchangers to dissipate heat [1-3]. Finite time thermodynamics (FTT) or entropy generation minimization (EGM) [15-29] concepts provide an approach to quantify cycles and plant component irreversibilities and thereafter can provide a more rationally based prescription applicable to engineering design and development process. Some authors have investigated the performances of thermoelectric refrigerators using a combination of finite time thermodynamics and non equilibrium thermodynamics. Vella et al. [30], Goktun [31], Xuan et al. [32], and Huang et al. [33] analyzed the effect of finite-rate heat transfer between the thermoelectric device and its external heat reservoirs on the performance of single-element thermoelectric refrigerators.

In practice, a commercial thermoelectric refrigerator is a multielement device, which is composed of many fundamental thermoelectric elements. Some researchers investigated the characteristics of multielement thermoelectric 
refrigerators with the irreversibilities of finite-rate heat transfer, Joulean heat, and the heat leakage through the thermoelectric elements. Considering the external irreversibilities of a thermoelectric refrigerator, much work has shown that the heat transfer irreversibility affects the performances of thermodynamic processes strongly. Xuan [34] derived a set of theoretical performance formulae from the thermal network model, based on which a general expression for the total cost of a thermoelectric refrigerator is presented. Riffat and Qiu [35] compared the performance of three types of domestic air-conditioners. Luo and Chen et al. [36, 37] optimized the ratio of the heat transfer surface area of the high temperature side heat exchanger to the total heat transfer surface area of the heat exchangers for maximizing the cooling load and the COP of a single- and two-stage thermoelectric refrigerators. Cheng and Lin [38] proposed a method of optimizing the dimensions of the thermoelectric elements legs using genetic algorithms to maximize the cooling load. Gurevich and Lashkevych [39, 40] investigated the physical peculiarities of the thermoelectric cooling. Chen et al. [41], Khattab and Shenawy [42], Chen et al. [43, 44] and Meng et al. [45-47] investigated the effectiveness and performance optimization of a single- or two-stage thermoelectric refrigerators (or heat pumps) driven by a single- or two-stage thermoelectric generators. Vian and Astrainet [48, 49] developed a domestic refrigerator with three compartments for food preservation.

Reviewing the former literatures concerning thermoelectric refrigerators, some features can be concluded as follows:

(1) Researches focusing the inner structure of a thermoelectric refrigerator, which are based on non equilibrium thermodynamics, are without considering the effects of external heat transfer irreversibility.

(2) Researches focusing the effects of external irreversibility of a thermoelectric refrigerator, which are based on finite time thermodynamics, are without considering the Thomson effect, inner structure and physical dimension of the thermoelectric refrigerator.

(3) Most of the researches are without considering the effects of the conducting trips, ceramic plate and especially the heat leakage through the air gap of the module.

To sum up, there is a lack of a complete model which can be applied to analyze the quantities of effects of inner and external factors on the performance of a commercial thermoelectric refrigerator with typical heat exchanger, and can be applied to predicate the performance of a practical commercially available thermoelectric module.

Taking inner and external multi-irreversibilities into account, this paper aims to establish a developed model of commercial thermoelectric refrigerators with a finned heat exchanger by introducing finite time thermodynamics. Numerical study and comparative investigation on the performance of a typical commercial thermoelectric refrigerator will be performed for cooling load and COP. The model and calculation method may be applied to not only the calculation, analysis and performance prediction of thermoelectric refrigerators, but also the design and optimization of the heat exchangers of the thermoelectric refrigerators.

\section{Analysis of a thermoelectric refrigerator and the developed model \\ 2.1. A commercial thermoelectric refrigerator}

Figure 1 shows a typical commercial thermoelectric refrigerator with external heat exchanger. The thermoelectric semiconductor legs are connected by copper conducting strips in serials. $P$-type and $N$-type semiconductor legs compose a basic thermoelectric element. The structure of the thermoelectric element and the connected conducting strip are shown in Fig. 2. The length and the side lengths of the cross-section of a semiconductor leg are $L, L_{a}$ and $L_{b}$, respectively. $L_{a}=L_{b}$ holds for a typical commercial thermoelectric element. The length and the thickness of the conducting strip are $L_{\mathrm{cu}}$ and $\delta_{\mathrm{cu}}$, respectively. The number of thermoelectric elements of the module is $N$. The conducting strips are fixed at a thermal conducting and electrical insulating ceramic plate. For a typical commercial thermoelectric module, the ceramic plate is square. The area and thickness of the ceramic plate are $A_{\mathrm{cp}}$ and $\delta_{\mathrm{cp}}$, respectively. The module is packaged by thermal insulation epoxy resin.

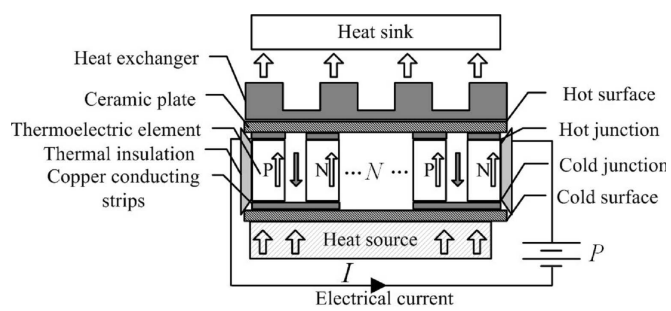

Fig. 1. Structure of a thermoelectric refrigerator with finned heat exchanger.

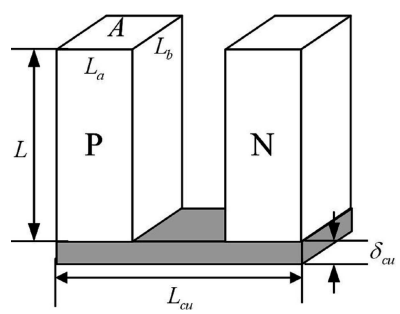

Fig. 2. Schematic diagram of a thermoelectric element.

\subsection{A finned heat exchanger}

A finned heat exchanger used for fluid heating or cooling is shown in Fig. 3. The base area, thickness and the side length of the exchanger are $A_{\mathrm{b}}, \delta_{\text {exb }}$ and $L_{\text {ex }}$, respectively. The height, length and thickness of the fin are $H_{\mathrm{f}}$, $L_{\mathrm{f}}$ and $\delta_{\mathrm{f}}$, respectively. The width of the channel is $\delta_{\mathrm{c}}$. $L_{\mathrm{ex}}=L_{\mathrm{f}}$ holds for a heat exchanger with square base. 


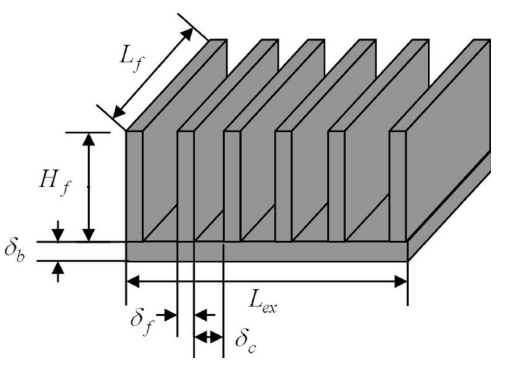

Fig. 3. Schematic diagram of a finned heat exchanger.

\subsection{Thermal resistance network}

The thermal resistance network of the whole device is shown in Fig. 4. The hot and cold junction temperatures of the thermoelectric elements are $T_{\mathrm{h}}$ and $T_{\mathrm{c}}$, respectively. The ceramic plate inner surface temperatures are $T_{\text {cpbh }}$ and $T_{\text {cpbc }}$, respectively. The ceramic plate exterior surface temperatures are $T_{\text {cpih }}$ and $T_{\text {cpic }}$, respectively. The fin base temperature is $T_{\text {exbh }}$. The heat sink (cooling water) and heat source temperatures (cooling object) are $T_{\mathrm{H}}$ and $T_{\mathrm{L}}$, respectively, where $T_{\mathrm{H}}=\left(T_{\mathrm{in}}+T_{\text {out }}\right) / 2$ and $T_{\mathrm{L}}=T_{\text {cpic }}$ for a water cooling and solid load device.

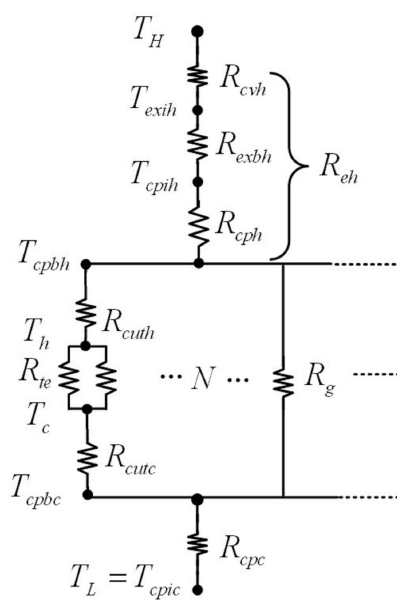

Fig. 4. Schematic diagram of the thermal resistance network.

The heat conduction thermal resistance of a thermoelectric element is $R_{\text {te }}=1 / K$, where $K$ is thermal conductance. The heat conduction thermal resistances of a conducting strip are $R_{\text {cuth }}$ and $R_{\text {cutc }}$, respectively. The heat conduction thermal resistance (the heat transfer through the air gap can be treated as heat conduction, which is approved in Appendix) of the air gap is $R_{\mathrm{g}}$. The heat conduction thermal resistances of the ceramic plate base are $R_{\mathrm{cph}}$ and $R_{\mathrm{cpc}}$, respectively. The heat conduction thermal resistance of the heat exchanger base is $R_{\text {exbh. }}$. The heat convection thermal resistance between the heat exchanger and the heat reservoir is $R_{\mathrm{cvh}}$.

The heat flow rate absorbed from the heat source to the module is $Q_{\mathrm{L}}$. The heat flow rate dissipated from the module to the heat $\operatorname{sink}$ is $Q_{\mathrm{H}}$. The heat flow rate through the hot and cold junctions of the thermoelectric elements are $Q_{\mathrm{h}}$ and $Q_{\mathrm{c}}$, respectively. The heat flow rate through the air gap is $Q_{\mathrm{g}}$.

\subsection{Electrical resistance network}

The electrical resistance network of the whole device is shown in Fig. 5. The electrical resistances of the $P$ and $N$-type semiconductor legs are $R_{p}$ and $R_{n}$, respectively. The electrical resistances of the hot and cold side conducting strip are $R_{\text {cuh }}$ and $R_{\text {cuc }}$, respectively. The input electrical voltage and input electrical current are $U$ and $I$, respectively.

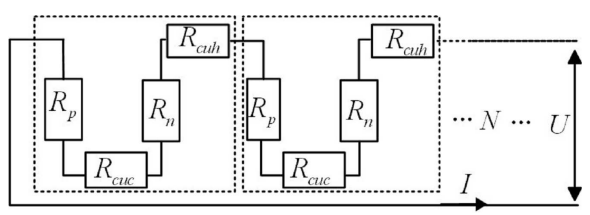

Fig. 5. Schematic diagram of the electrical resistance network.

\subsection{Energy equations}

For a well designed thermal insulation packaging module, the heat leakage through the surround of the module can be neglected. At steady working state, the temperature distribution of the air gap is the same with the thermoelectric elements, so the heat transfer of the whole device can be treated as one-dimensional.

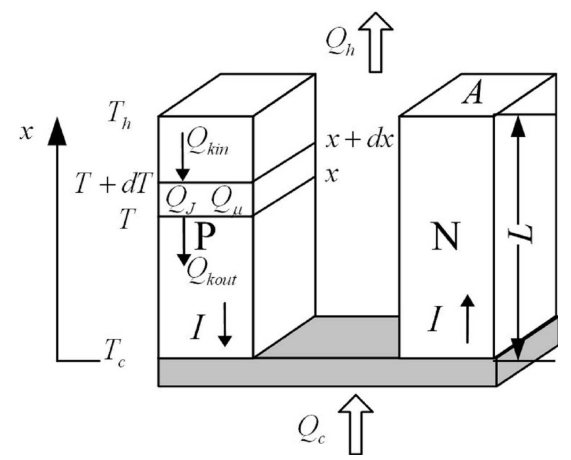

Fig. 6. Schematic diagram of the heat transfer of a thermoelectric element.

The infinitesimal as shown in Fig. 6 is considered. According to the non-equilibrium thermodynamics, the inner effects of the thermoelectric elements include the Seebeck effect, the Fourier effect, the Joule effect and the Thomson effect. The increment rate of inner energy of the infinitesimal is zero at steady-state, so one can obtain the energy conservation equation of $P$-type semiconductor leg as follows:

$$
Q_{\mathrm{Kin}}-Q_{\mathrm{Kout}}+Q_{\mathrm{J}}+Q_{\mu}=0,
$$

where $Q_{\mathrm{Kin}}, Q_{\mathrm{Kout}}, Q_{\mathrm{J}}$ and $Q_{\mu}$ are the Fourier heat input, the Fourier heat output, generated Joule heat and 
the Thomson heat. Equation (1) can be written as

$$
\begin{aligned}
& \frac{\mathrm{d}}{\mathrm{d} x}\left(T_{p}+\mathrm{d} T_{p}\right) k_{p} A_{p}-\frac{\mathrm{d} T_{p}}{\mathrm{~d} x} k_{p} A_{p}+\left(e J_{p}\right)^{2} \frac{A_{p} L_{p}}{\sigma_{p}} \frac{\mathrm{d} x}{L_{p}} \\
& \quad+\mu_{p} I \mathrm{~d} T_{p}=0,
\end{aligned}
$$

where $k_{p}, \sigma_{p}, \mu_{p}, A_{p}, L_{p}, T_{p}, e J_{p}$ are the thermal conductivity, electrical conductivity, Thomson coefficient, cross section area, length, temperature and electrical current density of the $P$-type semiconductor leg. Reforming Eq. (2) and making the same analysis on the $N$-type semiconductor leg, one can obtain the heat conduction differential Eqs. (3) and (4) with boundary conditions (5) and (6) as follows [9]:

$$
\begin{aligned}
& \frac{\mathrm{d}}{\mathrm{d} x}\left(k_{p} \frac{\mathrm{d} T_{p}}{\mathrm{~d} x}\right)-e J_{p} \mu_{p} \frac{\mathrm{d} T_{p}}{\mathrm{~d} x}+\frac{\left(e J_{p}\right)^{2}}{\sigma_{p}}=0, \\
& \frac{\mathrm{d}}{\mathrm{d} x}\left(k_{n} \frac{\mathrm{d} T_{n}}{\mathrm{~d} x}\right)+e J_{n} \mu_{n} \frac{\mathrm{d} T_{n}}{\mathrm{~d} x}+\frac{\left(e J_{n}\right)^{2}}{\sigma_{n}}=0, \\
& T_{p}(0)=T_{n}(0)=T_{\mathrm{c}}, \\
& T_{p}\left(L_{p}\right)=T_{n}\left(L_{n}\right)=T_{\mathrm{h}},
\end{aligned}
$$

where $k_{n}, \sigma_{n}, \mu_{n}, T_{n}, e J_{n}$ are the thermal conductivity, electrical conductivity, Thomson coefficient, temperature and electrical current density of the $N$-type semiconductor leg.

Taking into account the effect of temperature dependence of thermoelectric properties, $k_{p}, \sigma_{p}$ and $\mu_{p}$ are function of $T_{p}$; while $k_{n}, \sigma_{n}$ and $\mu_{n}$ are function of $T_{n}$. However, such a differential equation cannot be solved analytically. Replacing $k, \sigma$ and $\mu$ with the mean value $\bar{k}, \bar{\sigma}$ and $\bar{\mu}$ approximately gives approximation of Eqs. (3) and (4) as follows:

$$
\begin{aligned}
& \overline{k_{p}} A_{p} \frac{\mathrm{d} T_{p}^{2}}{\mathrm{~d} x^{2}}-\overline{\mu_{p}} I \frac{\mathrm{d} T_{p}}{\mathrm{~d} x}+\frac{I^{2}}{\overline{\sigma_{p}} A_{p}}=0, \\
& \overline{k_{n}} A_{n} \frac{\mathrm{d} T_{n}^{2}}{\mathrm{~d} x^{2}}+\overline{\mu_{n}} I \frac{\mathrm{d} T_{n}}{\mathrm{~d} x}+\frac{I^{2}}{\overline{\sigma_{n}} A_{n}}=0,
\end{aligned}
$$

where $\overline{k_{p}}=\left.k_{p}\right|_{T=\left(T_{\mathrm{h}}+T_{\mathrm{c}}\right) / 2}, \quad \overline{\sigma_{p}}=\left.\sigma_{p}\right|_{T=\left(T_{\mathrm{h}}+T_{\mathrm{c}}\right) / 2}$, $\overline{\mu_{p}}=\left.\mu_{p}\right|_{T=\left(T_{\mathrm{h}}+T_{\mathrm{c}}\right) / 2}$ for $P$-type semiconductor leg and $\overline{k_{n}}=\left.k_{n}\right|_{\mathrm{T}=\left(\mathrm{T}_{\mathrm{h}}+\mathrm{T}_{\mathrm{c}}\right) / 2}, \overline{\sigma_{n}}=\left.\sigma_{n}\right|_{\mathrm{T}=\left(\mathrm{T}_{\mathrm{h}}+\mathrm{T}_{\mathrm{c}}\right) / 2}, \overline{\mu_{n}}=$

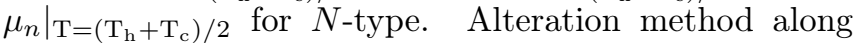
with the following fitted formulae Eqs. (44)-(46) should be adopted to determine the junction temperatures $T_{\mathrm{h}}$ and $T_{\mathrm{c}}$.

The net heat flow rate through a thermoelectric element junction is the Peltier heat minus the Fourier heat (heat leakage). One can obtain the total heat flow rates through the hot and cold junctions as follows:

$$
\begin{aligned}
Q_{\mathrm{h}} & =N\left(\left(\alpha_{p \mathrm{~h}}-\alpha_{n \mathrm{~h}}\right) T_{\mathrm{h}} I-\left.\overline{k_{p}} A_{p} \frac{\mathrm{d} T_{p}}{\mathrm{~d} x}\right|_{x=L_{p}}\right. \\
& \left.-\left.\overline{k_{n}} A_{n} \frac{\mathrm{d} T_{n}}{\mathrm{~d} x}\right|_{x=L_{n}}\right), \\
Q_{\mathrm{c}} & =N\left(\left(\alpha_{p \mathrm{c}}-\alpha_{n \mathrm{c}}\right) T_{\mathrm{c}} I\right.
\end{aligned}
$$

$$
\left.-\left.\overline{k_{p}} A_{p} \frac{\mathrm{d} T_{p}}{\mathrm{~d} x}\right|_{x=0}-\left.\overline{k_{n}} A_{n} \frac{\mathrm{d} T_{n}}{\mathrm{~d} x}\right|_{x=0}\right),
$$

where $\alpha_{p}$ and $\alpha_{n}$ are the Seebeck coefficient of the $P$ - and $N$-type semiconductor leg, and the subscripts $\mathrm{h}$ and c represent the hot and cold side.

The heat flow rates through the conducting strips are given by

$$
\begin{aligned}
& Q_{\mathrm{h} i}=N\left(T_{\mathrm{h}}-T_{\mathrm{cpbh}}\right) / R_{\text {cuth }}, \\
& Q_{\mathrm{c} i}=N\left(T_{\mathrm{cpbc}}-T_{\mathrm{c}}\right) / R_{\text {cutc }} .
\end{aligned}
$$

It is assumed that the total thermal resistance between the hot side ceramic plate inner surface and the heat sink is $R_{\mathrm{eh}}=R_{\mathrm{cvh}}+R_{\mathrm{exbh}}+R_{\mathrm{cph}}$; the total thermal resistance between the cold side ceramic plate inner surface and the heat sourceis $R_{\mathrm{ec}}=R_{\mathrm{cpc}}$. Then, one has

$$
\begin{aligned}
& Q_{\mathrm{H}}=\left(T_{\mathrm{cpbh}}-T_{\mathrm{H}}\right) / R_{\mathrm{eh}}, \\
& Q_{\mathrm{L}}=\left(T_{\mathrm{L}}-T_{\mathrm{cpbc}}\right) / R_{\mathrm{ec}} .
\end{aligned}
$$

The heat flow rate through the air gap (the gray arrows in Fig. 1) is

$$
Q_{\mathrm{g}}=\left(T_{\mathrm{cpbh}}-T_{\mathrm{cpbc}}\right) / R_{\mathrm{g}} .
$$

Equation (15) is an improvement of this paper beyond traditional literatures. The following analysis will prove that the heat transfer through the air gap should be considered because it affects the performance of the device strongly. The control equations i.e. the relations of heat flow rates can be expressed as follows:

$$
\begin{aligned}
& Q_{\mathrm{H}}=Q_{\mathrm{h}}-Q_{\mathrm{g}}, \\
& Q_{\mathrm{h}}=Q_{\mathrm{h} i}, \\
& Q_{\mathrm{c}}=Q_{\mathrm{c} i}, \\
& Q_{\mathrm{L}}=Q_{\mathrm{c}}-Q_{\mathrm{g}} .
\end{aligned}
$$

\section{Solution of the model}

\subsection{Solution of the thermoelectric element}

The temperature distribution of the $P$ - and $N$-type semiconductor leg can be obtained by solving Eqs. (7) and (8) as follows:

$$
\begin{aligned}
& T_{p}(x)=T_{\mathrm{c}}-F_{p} x+\frac{T_{\mathrm{h}}-T_{\mathrm{c}}+F_{p} L_{p}}{\left(\mathrm{e}^{\omega_{p} L_{p}}-1\right)}\left(\mathrm{e}^{\omega_{p} x}-1\right), \\
& T_{n}(x)=T_{\mathrm{c}}+F_{n} x+\frac{T_{\mathrm{h}}-T_{\mathrm{c}}-F_{n} L_{n}}{\left(\mathrm{e}^{-\omega_{n} L_{n}}-1\right)}\left(\mathrm{e}^{-\omega_{n} x}-1\right),
\end{aligned}
$$

where $\omega_{p}=\overline{\mu_{p}} I /\left(\overline{k_{p}} A_{p}\right), \quad F_{p}=I /\left(\overline{\sigma_{p} \mu_{p}} A\right), \omega_{n}=$ $\overline{\mu_{n}} I /\left(\overline{k_{n}} A_{n}\right)$ and $F_{n}=I /\left(\overline{\sigma_{n} \mu_{n}} A\right)$.

For maximum figure of merit of the thermoelectric element $Z=\alpha^{2} / K R$, the dimension of the thermoelectric element and the physical property of the material should satisfy the following equation [2]: 


$$
\frac{A_{p}^{2} L_{n}^{2}}{A_{n}^{2} L_{p}^{2}}=\frac{k_{n} \sigma_{n}}{k_{p} \sigma_{p}}
$$

To reduce the cost of manufacture, the $P$ - and $N$-type semiconductor legs are made with same dimensions i.e. $A_{p}=A_{n}=A$ and $L_{p}=L_{n}=L$. So one has $k_{n} \sigma_{n} /\left(k_{p} \sigma_{p}\right)=1$ by Eq. (22). Similar doped alloys are adopted to make $P$ - and $N$-type semiconductor legs. That is $\sigma_{p}=\sigma_{n}=\sigma, k_{p}=k_{n}=k, \alpha_{p}=-\alpha_{n}, \mu_{p}=-\mu_{n}$. According to Taylor's formula, when $|x| \ll 1$, $\mathrm{e}^{x} \approx 1+x$ holds true. Based on above assumption, Eqs. (9) and (10) can be approximated as follows at the case of $\bar{\mu} I / K \ll 1$ :

$$
\begin{aligned}
& Q_{\mathrm{h}}=N\left[\alpha_{\mathrm{h}} I T_{\mathrm{h}}-K\left(T_{\mathrm{h}}-T_{\mathrm{c}}\right)+0.5 I^{2} R\right. \\
& \left.\quad-0.5 \bar{\mu} I\left(T_{\mathrm{h}}-T_{\mathrm{c}}\right)\right], \\
& Q_{\mathrm{c}}=N\left[\alpha_{\mathrm{c}} I T_{\mathrm{c}}-K\left(T_{\mathrm{h}}-T_{\mathrm{c}}\right)-0.5 I^{2} R\right. \\
& \left.\quad+0.5 \bar{\mu} I\left(T_{\mathrm{h}}-T_{\mathrm{c}}\right)\right],
\end{aligned}
$$

where $\alpha_{\mathrm{h}}=\alpha_{\mathrm{h} p}-\alpha_{\mathrm{h} n}$ and $\alpha_{\mathrm{c}}=\alpha_{\mathrm{cp}}-\alpha_{c n}$ are the Seebeck coefficient of the thermoelectric elements at hot and cold side. $\bar{\mu}=\overline{\mu_{p}}-\overline{\mu_{n}}=2 \overline{\mu_{p}}, K$ and $R$ are the total Thomson coefficient, thermal conductance and electrical resistance of a thermoelectric element. $\alpha I T, K \Delta T, I^{2} R$ and $\bar{\mu} I \Delta T$ are the rates of the Peltier heat, the Fourier heat, the Joule heat and the Thomson heat, respectively. $K$ and $R$ are given by

$$
\begin{aligned}
K & =K_{p}+K_{n}=\overline{k_{p}} A_{p} / L_{p}+\overline{k_{n}} A_{n} / L_{n} \\
& =2 \bar{k} A / L, \\
R & =R_{p}+R_{n}=L_{p} /\left(\overline{\sigma_{p}} A_{p}\right)+L_{n} /\left(\overline{\sigma_{n}} A_{n}\right) \\
& =2 L /(\bar{\sigma} A) .
\end{aligned}
$$

The power input is difference between the absorbed and dissipated heat flow rate and results in

$$
\begin{aligned}
P & =Q_{\mathrm{h}}-Q_{\mathrm{c}}=N I\left[\alpha_{\mathrm{h}} T_{\mathrm{h}}-\alpha_{\mathrm{c}} T_{\mathrm{c}}+I R\right. \\
& \left.-\bar{\mu}\left(T_{\mathrm{h}}-T_{\mathrm{c}}\right)\right] .
\end{aligned}
$$

Taking the Joule heat generated by the conducting trips as the thermoelectric elements generated and replacing $R$ with $R_{\mathrm{t}}=R+R_{\mathrm{cu}}$ gives the modified input power as follows:

$$
\begin{aligned}
P & =Q_{\mathrm{h}}-Q_{\mathrm{c}}=N I\left[\alpha_{\mathrm{h}} T_{\mathrm{h}}-\alpha_{\mathrm{c}} T_{\mathrm{c}}+I R_{\mathrm{t}}\right. \\
& \left.-\bar{\mu}\left(T_{\mathrm{h}}-T_{\mathrm{c}}\right)\right] .
\end{aligned}
$$

The coefficient of performance $\mathrm{COP}=Q_{\mathrm{L}} / P$ is given by

$$
\mathrm{COP}=\frac{\left(T_{\mathrm{L}}-T_{\mathrm{cpbc}}\right) / R_{\mathrm{ec}}}{N I\left[\alpha_{\mathrm{h}} T_{\mathrm{h}}-\alpha_{\mathrm{c}} T_{\mathrm{c}}+I R_{\mathrm{t}}-\bar{\mu}\left(T_{\mathrm{h}}-T_{\mathrm{c}}\right)\right]} .
$$

\subsection{Calculation method of the thermal and electrical resistances}

The heat conduction thermal resistance of a conducting strip and a ceramic plate are

$$
R_{\text {cut }}=\delta_{\text {cu }} / k_{\text {cu }} A,
$$

$$
R_{\mathrm{cp}}=\delta_{\mathrm{cp}} / k_{\mathrm{cp}} A_{\mathrm{cp}} .
$$

The heat conduction thermal resistance of the heat exchanger base is

$$
R_{e x b}=\delta_{\mathrm{b}} / k_{\mathrm{ex}} A_{\mathrm{b}} .
$$

According to the heat transfer theory, the heat convection thermal resistance between the finned heat exchanger and the heat reservoir is [50]:

$$
R_{\mathrm{cv}}=\frac{1}{h_{\mathrm{cv}} A_{\mathrm{eff}}},
$$

where $h_{\mathrm{cv}}$ and $A_{\text {eff }}$ are the coefficient of heat convection and the effective heat transfer area. $h_{\mathrm{cv}}$ can be calculated by

$$
h_{\mathrm{cv}}=\frac{\mathrm{Nu} k_{\mathrm{f}}}{H_{\mathrm{f}}},
$$

where $\mathrm{Nu}, k_{\mathrm{f}}$ and $H_{\mathrm{f}}$ are the Nusselt number of the flow, thermal conductivity of the fluid and the height of the fins. $\mathrm{Nu}$ can be calculated by [50]:

$$
\mathrm{Nu}=0.664 \mathrm{Re}^{1 / 2} \operatorname{Pr}_{\mathrm{f}}^{1 / 3}, \quad \operatorname{Re}<5 \times 10^{5}
$$

(lamellar flow)

$$
\mathrm{Nu}=0.037 \operatorname{Re}^{4 / 5} \operatorname{Pr}_{\mathrm{f}}^{1 / 3}, \quad \operatorname{Re} \geq 5 \times 10^{5}
$$

(turbulent flow)

where $\operatorname{Pr}_{f}$ and $R e$ are the Prandtl number of the fluid and the Reynolds number of the flow. They can be calculated by

$$
\begin{aligned}
& \operatorname{Pr}_{\mathrm{f}}=\frac{v_{\mathrm{f}}}{a_{\mathrm{f}}}=\frac{c_{\mathrm{pf}} \varphi_{\mathrm{f}}}{k_{\mathrm{f}}}, \\
& \operatorname{Re}=v_{\mathrm{f}} H_{\mathrm{f}} / v_{\mathrm{f}},
\end{aligned}
$$

where $v_{\mathrm{f}}, a_{\mathrm{f}}, c_{\mathrm{pf}}, k_{\mathrm{f}}, \varphi_{\mathrm{f}}, \rho_{\mathrm{f}}$ and $v_{\mathrm{f}}$ are the kinematic viscosity, thermal diffusivity, constant pressure heat capacity ratio, thermal conductivity, kinetic velocity, density and the viscosity of the fluid, respectively.

The effective heat transfer area is given by

$$
A_{\text {eff }}=\eta_{\mathrm{f}}\left(2 N_{\mathrm{f}} L_{\mathrm{f}} H_{\mathrm{f}}\right)+\left(N_{\mathrm{f}}-1\right) \delta_{\mathrm{c}} L_{\mathrm{f}},
$$

where the fin efficiency $\eta_{\mathrm{f}}$ is given by

$$
\eta_{\mathrm{f}}=\frac{\tanh \left(m H_{\mathrm{f}}\right)}{m H_{\mathrm{f}}}
$$

where

$$
m=\sqrt{\frac{2 h_{\mathrm{cv}}\left(\delta_{\mathrm{f}}+L_{\mathrm{f}}\right)}{k_{\mathrm{f}} \delta_{\mathrm{f}} L_{\mathrm{f}}}} .
$$

To describe the geometry of the thermoelectric module air gap, a ratio is introduced from Ref. [51] and defined as module packed density $\theta$ :

$$
\theta=2 A N / A_{\mathrm{cp}} .
$$

The electrical resistance of a conducting strip is given as follows: 


$$
\begin{aligned}
R_{\mathrm{cu}} & =\frac{1}{\sigma_{\mathrm{cu}}} \frac{L_{\mathrm{cu}}}{\delta_{\mathrm{cu}} L_{b}}=\frac{1}{\sigma_{\mathrm{cu}}} \frac{2 L_{a}+L_{a}(1-\theta) / \theta}{\delta_{\mathrm{cu}} L_{b}} \\
= & \frac{1}{\sigma_{\mathrm{cu}}} \frac{1+1 / \theta}{\delta_{\mathrm{cu}}},
\end{aligned}
$$

where $\sigma_{\mathrm{cu}}$ and $\delta_{\mathrm{cu}}$ are the electrical conductivity and thickness of the conducting strip.

The heat transfer through the air gap of the module is treated as heat conduction (see Appendix) and then the thermal resistance is

$$
R_{\mathrm{g}}=\frac{L}{A_{\mathrm{g}} k_{\mathrm{air}}}=\frac{L}{A_{\mathrm{cp}} k_{\mathrm{air}}(1-\theta)} .
$$

Based on above calculation method, the performance of a fluid-cooling thermoelectric refrigerator with finned heat exchanger can be predicted exactly and roundly.

\section{Numerical simulation}

Numerical studies on the thermoelectric refrigerator performance including cooling load and COP for cooling solid object are performed. The physical properties of the commercially available material by Melcor [52] used for this simulation are shown as follows:

$$
\begin{aligned}
& \alpha_{p}=\left(22224.0+930.6 T-0.9905 T^{2}\right) 10^{-9} \mathrm{~V} \mathrm{~K}^{-1},(44) \\
& \rho=\left(5112.0+163.4 T+0.6279 T^{2}\right) 10^{-10} \Omega \mathrm{m}, \quad(45) \\
& k=\left(62605.0-277.7 T+0.4131 T^{2}\right) 10^{-4} \mathrm{~W} \mathrm{~m}^{-1} \mathrm{~K}^{-1},
\end{aligned}
$$

where $\alpha_{p}, \rho$ and $k$ are the Seebeck coefficient, electrical resistivity and thermal conductivity. $T=\left(T_{\mathrm{h}}+T_{\mathrm{c}}\right) / 2$ is the mean temperature of the hot and cold junction.

The physical dimension of the thermoelectric module made by Ferrotec (9501/127/030B) [53] are shown in Table I. The physical properties of the conducting trip, ceramic plate, air, and heat exchanger are shown in Table II [52]. The heat exchanger is made of aluminum and the physical dimensions are shown in Table III. Parameters of cooling-water are shown in Table IV [54].

TABLE I

Dimensions of the thermoelectric module (Ferrotec 9501/ 127/030B) [53].

\begin{tabular}{c|c|c|c|c}
\hline \hline$A\left[\mathrm{~mm}^{2}\right]$ & $L[\mathrm{~mm}]$ & $\delta_{\mathrm{cu}}[\mathrm{mm}]$ & $\delta_{\mathrm{cp}}[\mathrm{mm}]$ & $A_{\mathrm{cp}}\left[\mathrm{mm}^{2}\right]$ \\
\hline 1 & 1.9 & 0.2 & 0.9 & $29.7 \times 29.7$
\end{tabular}

\section{TABLE II}

Physical properties of conducting strip, ceramic plate, air, and heat exchanger [52].

\begin{tabular}{c|c|c|c|c}
\hline \hline $\begin{array}{c}\rho_{\mathrm{cu}} \\
\Omega \mathrm{m}]\end{array}$ & $k_{\mathrm{cu}}$ & $k_{\mathrm{cp}}$ & $\begin{array}{c}k_{\mathrm{air}} \\
{\left[\mathrm{W} \mathrm{m}^{-1} \mathrm{~K}^{-1}\right]}\end{array}$ & $k_{\mathrm{Al}}$ \\
\hline $1.7 \times 10^{-8}$ & 386 & 35.3 & $2.57 \times 10^{-2}$ & 204
\end{tabular}

Dimensions of the heat exchanger.

\begin{tabular}{c|c|c|c|c|c}
\hline \hline$A_{\mathrm{b}}\left[\mathrm{mm}^{2}\right]$ & $\delta_{\mathrm{b}}[\mathrm{mm}]$ & $\delta_{\mathrm{f}}[\mathrm{mm}]$ & $\delta_{\mathrm{c}}[\mathrm{mm}]$ & $H_{\mathrm{f}}[\mathrm{mm}]$ & $N_{\mathrm{f}}$ \\
\hline $29.7 \times 29.7$ & 2 & 1.3 & 1.3 & 20 & 12
\end{tabular}

Parameters of cooling water [54].

TABLE IV

\begin{tabular}{c|c|c|c|c}
\hline \hline $\operatorname{Pr}_{\text {water }}$ & $\begin{array}{c}k_{\text {water }} \\
{\left[\mathrm{W} \mathrm{m}^{-1} \mathrm{~K}^{-1}\right]}\end{array}$ & $\begin{array}{c}v \\
{\left[\mathrm{~m} \mathrm{~s}^{-1}\right]}\end{array}$ & $\begin{array}{c}T_{\mathrm{H}} \\
{[\mathrm{K}]}\end{array}$ & $\begin{array}{c}T_{\mathrm{L}} \\
{[\mathrm{K}]}\end{array}$ \\
\hline 7.02 & $59.9 \times 10^{-2}$ & 0.5 & 300 & 290
\end{tabular}

\subsection{Irreversibility analysis}

Figure 7 shows cooling load and COP versus input electrical current of different models. $A=1 \mathrm{~mm}^{2}$, $L=1.9 \mathrm{~mm}, N=127$ and $\Delta T=10 \mathrm{~K}$ are set in the figure. Curve 1 represents the result of the exo-reversible model, in which all external irreversibilities are neglected. Curve 2 represents the result of the model, in which the heat transfer through the air gap is considered. Curve 3 represents the result of the model, in which the heat convection between the heat exchanger and the water is considered. Curve 4 represents the result of the irreversible model, in which the multiform external thermal resistances, including the thermal resistance of conducting strips, ceramic plates, heat exchanger base and heat convection, are considered. One can see that the heat convection of heat exchanger and the heat leakage through the air gap decrease the cooling load and COP heavily. However, the heat leakage through the air gap is neglected in traditional literatures. For any model of the thermoelectric refrigerator, there is one optimal input electrical current $I^{\mathrm{COP}}$ corresponding to the maximum cooling load $Q_{\mathrm{L}, \max }$ and another optimal input electrical current $I^{Q}$ corresponding to the maximum COP, $\mathrm{COP}_{\max }$. This is a basic characteristic of a thermoelectric refrigerator which cannot be changed by the external irreversibility. However, because of the multiform external irreversibility, $I^{Q}$ decreases a lot while $I^{\mathrm{COP}}$ retains constant approximately. The numerical calculations show that although the thermal resistance of conducting strip, ceramic plate, heat exchanger base and the electrical resistance of the conducting strips would decrease the performance of the device to different extent, the effects are small comparing with the heat convection of heat exchanger and the heat leakage through the air gap.

For the exo-reversible model, the maximum cooling load and the maximum COP are $3.55 \mathrm{~W}$ and 0.73 ; for the irreversible model, the maximum cooling load and the maximum COP are $2.33 \mathrm{~W}$ and 0.54 . That is the multi-irreversibilities decreased the maximum cooling load and the maximum COP by $34 \%$ and $26 \%$, respectively.

\subsection{Effects of several important parameters}

The performances of the thermoelectric refrigerator depend on many factors of the device such as physical prop- 


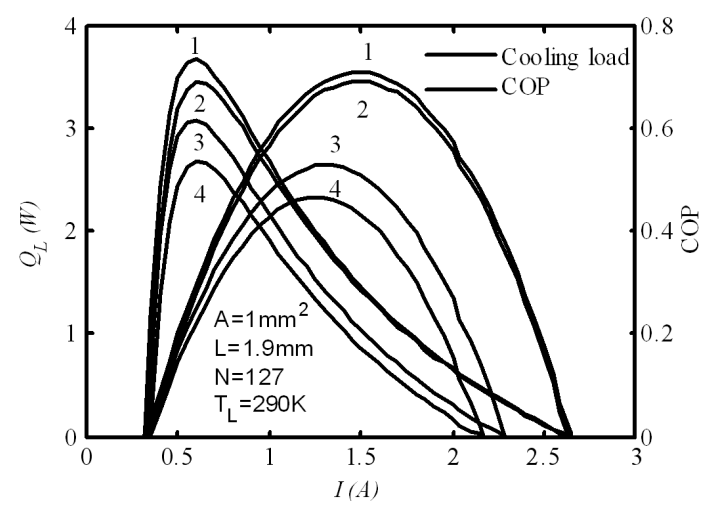

Fig. 7. Cooling load and COP versus input electrical current characteristic of different models.

erties, geometrical dimensions, temperature parameters and flow parameters. According to the quantity of effect on the performance of the device, the effects of several important parameters i.e. the cooling temperature difference $\Delta T$, the length of the thermoelectric elements $L$, the cross-section of the thermoelectric elements $A$, and the heat exchanger base area $A_{\mathrm{b}}$ are analyzed, respectively.

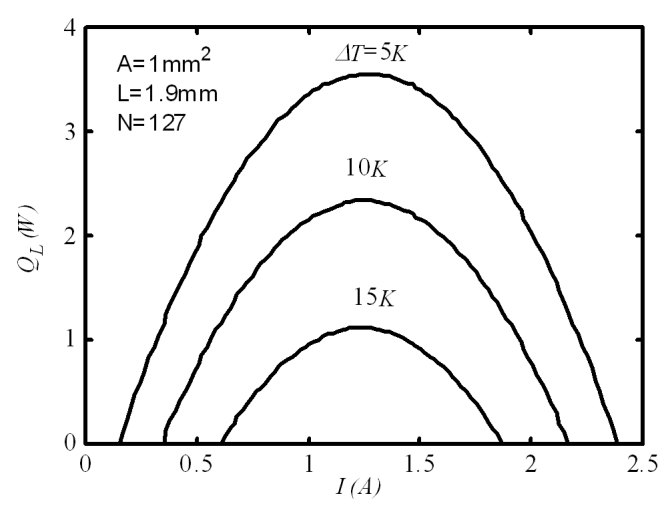

Fig. 8. Effects of cooling temperature difference on cooling load versus input electrical current.

Figures 8 and 9 show the effects of cooling temperature difference $\Delta T=T_{\mathrm{H}}-T_{\mathrm{L}}$ on cooling load and COP versus input electrical current, respectively. $A=1 \mathrm{~mm}^{2}$, $L=1.9 \mathrm{~mm}$ and $N=127$ are set in the figures. It can be seen that with the increase in cooling temperature difference, both the cooling load and the COP decrease rapidly, that is, the thermoelectric refrigerator is not suitable for large cooling temperature difference for its excessive performance decline and diseconomy. As a consequence, the optimal input electrical current $I^{Q}$ corresponding to the maximum COP increases while the optimal input electrical current $I^{\mathrm{COP}}$ corresponding to the maximum cooling load remains constant approximately.

Figures 10 and 11 show the effects of length of thermoelectric elements $L$ on the cooling load and COP versus input electrical current characteristics, respectively.

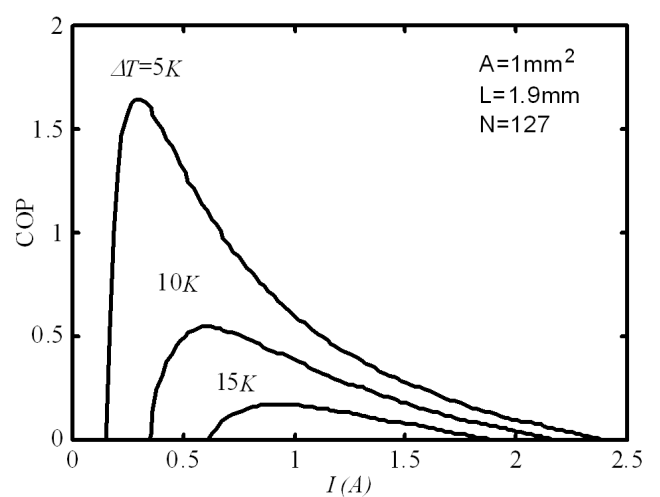

Fig. 9. Effects of cooling temperature difference on the COP versus input electrical current.

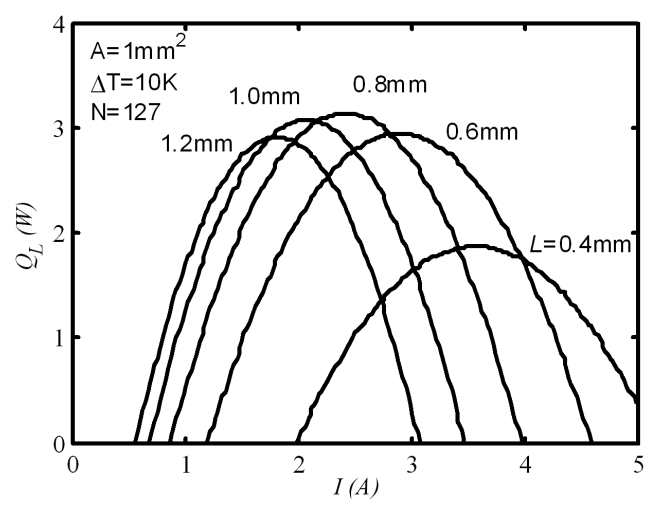

Fig. 10. Effects of length of thermoelectric elements on the cooling load versus input electrical current.

$A=1 \mathrm{~mm}^{2}, \Delta T=10 \mathrm{~K}$ and $N=127$ are set in the figures. It can be seen that the maximum cooling load increases and then decreases with the increase in length of thermoelectric elements, that is, there is an optimal length of thermoelectric elements $L^{Q}$ corresponding to the maximum cooling load. In this case, the optimal

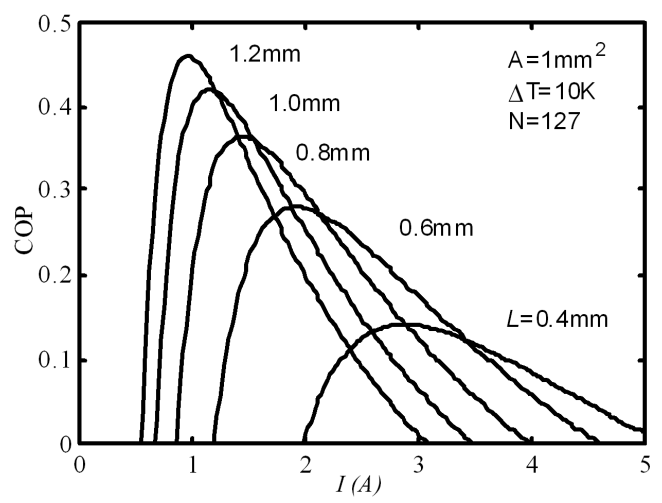

Fig. 11. Effects of length of thermoelectric elements on the COP versus input electrical current. 
length of thermoelectric elements is about $0.8 \mathrm{~mm}$. With the increase in length of thermoelectric elements, the COP increase monotonously. In application to the geometry design of practical thermoelectric refrigerators, appropriate length of thermoelectric elements should be selected from the point of view of finite time thermodynamic optimization (the compromise optimization between cooling load and the COP). The length of thermoelectric elements also affects the optimal input electrical current. With the increase in length of thermoelectric elements, both the optimal input electrical currents corresponding to the maximum cooling load and to the maximum COP decrease.

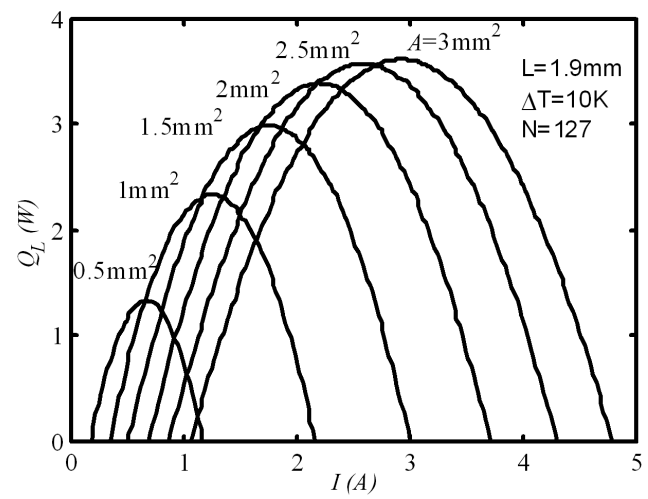

Fig. 12. Effects of cross-section area of thermoelectric elements on the cooling load versus input electrical current.

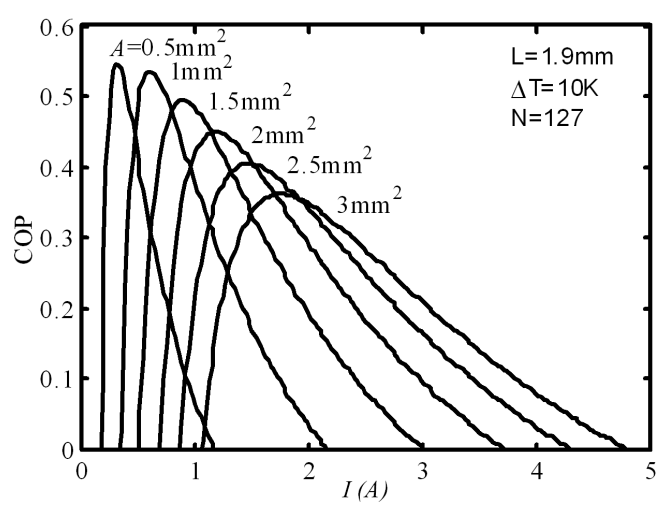

Fig. 13. Effects of thermoelectric cross-section area on the COP versus input electrical current.

Figures 12 and 13 show the effects of cross-section area of thermoelectric elements $A$ on the cooling load and COP versus input electrical current, respectively. In the calculations, $\Delta T=10 \mathrm{~K}, L=1.9 \mathrm{~mm}$ and $N=127$ holds. The packed density area $\theta=2 A / A_{\mathrm{cp}}$ corresponding to different cross-section area are 0.1440 , $0.2880,0.4319,0.5759,0.7199,0.8639$, respectively. It can be seen that the maximum cooling load increases rapidly while the maximum COP decreases slowly with the increase in cross-section area of thermoelectric elements. Surely, there is a limit of cooling load by increasing the cross-section area of thermoelectric elements because $\theta \leq 1$. In application to the design of practical thermoelectric refrigerators, the cooling load can be improved by increasing cross-section area of thermoelectric elements appropriately. It should be noted that the cost is that the COP will decrease a little and the device needs larger input electrical current and more thermoelectric materials.

TABLE V

Effect of the heat exchanger base area on the optimal performance.

\begin{tabular}{c|c|c|c|c}
\hline \hline$A_{\mathrm{b}} / A_{\mathrm{m}}$ & $I^{Q}[\mathrm{~A}]$ & $Q_{\mathrm{L} \max }[\mathrm{W}]$ & $I^{\mathrm{COP}}[\mathrm{A}]$ & $\mathrm{COP}_{\max }$ \\
\hline 0.5 & 1.11 & 1.7737 & 0.59 & 0.4590 \\
1.0 & 1.23 & 2.2823 & 0.59 & 0.5386 \\
2.0 & 1.29 & 2.6277 & 0.59 & 0.5872 \\
3.0 & 1.33 & 2.7670 & 0.59 & 0.6057 \\
4.0 & 1.37 & 2.9516 & 0.59 & 0.6294 \\
4.5 & 1.37 & 2.9668 & 0.59 & 0.6313 \\
5.0 & 1.37 & 2.9794 & 0.59 & 0.6329
\end{tabular}

The numerical calculations show that among the dimensions of the heat exchanger the heat exchanger base area $A_{\mathrm{b}}$ has greatest effect on the performance of the device. Table $\mathrm{V}$ lists the maximum cooling load $Q_{\mathrm{L} \text { max }}$, the maximum $\mathrm{COP}, \mathrm{COP}_{\max }$ and the corresponding electrical current $I^{Q}, I^{\mathrm{COP}}$ with different heat exchanger base area $A_{\mathrm{b}}$. It can be seen that the heat exchanger base area does affect the optimal performance of the device. With the increase in the heat exchanger base area, the maximum cooling load $Q_{\mathrm{L} \max }$, the corresponding optimal electrical current $I^{Q}$ and the maximum COP, COP $\max$, increase while the optimal electrical current $I^{\mathrm{COP}}$ corresponding to the maximum COP remains constant. This is a feature in the analysis of the optimal electrical current. Moreover, the less heat area of the exchanger base, the more effect on the optimal performance. When the heat exchanger base area is large enough, the optimal performance and the corresponding electrical current increase a little. The calculations show that when the area of the heat exchanger base is 3 times as large as the area of the ceramic plate of the thermoelectric refrigerator, the maximum cooling load and maximum COP is $95.89 \%$ and $92.88 \%$ of the performance limits, respectively. This conclusion can be reference for the design or selection of heat exchanger for thermoelectric refrigerators.

\section{Conclusions}

Taking inner and external multi-irreversibilities into account, a developed model of commercial thermoelectric refrigerator with finned heat exchanger is built by combining finite time thermodynamics with non-equilibrium 
thermodynamics. The model includes physical properties, geometrical dimension, temperature parameters and flow parameters. Numerical studies and comparative investigation on the performance of a typical commercial water-cooling thermoelectric refrigerator, which consists of 127 thermoelectric elements, show that the maximum cooling load is $2.33 \mathrm{~W}$ and maximum COP of the refrigerator is 0.54 when the cooling temperature difference is $10 \mathrm{~K}$. The multi-irreversibilities decreased the maximum cooling load and the maximum COP by $34 \%$ and $26 \%$, respectively. The irreversibility analysis show that there are many factors causing irreversibilities. Sorting by the quantity of effect on the performance of the device, the maximum is the thermal resistance of the heat convection between the heat exchanger and the cooling-water, followed by the heat leakage through the air gap of the thermoelectric module, ceramic plates, and thermal resistance of the heat exchanger base, the minima are thermal and electrical resistance of the conducting strips. The performance of the device can be improved by optimizing the length and cross-section area of the thermoelectric elements. When the area of the heat exchanger base is 3 times as large as the area of the ceramic plate of the thermoelectric refrigerator, the maximum cooling load and maximum COP is $95.89 \%$ and $92.88 \%$ of the performance limits, respectively. The model and calculation method can be applied to not only the performance prediction of thermoelectric refrigerators, but also the optimization of the heat exchanger of thermoelectric refrigerators.

\section{Acknowledgments}

This paper is supported by the National Natural Science Foundation of P.R. China (project No. 10905093), Program for New Century Excellent Talents in University of P.R. China (project No. NCET-04-1006) and the Foundation for the Author of National Excellent Doctoral Dissertation of P.R. China (project No. 200136). The authors wish to thank the reviewers for their careful, unbiased and constructive suggestions, which led to this revised manuscript.

\section{Appendix}

According to the heat transfer theory, the air gap of the module can be considered as a restricted horizontal interlayer. There are two cases: when the underside is cold side, the heat transfer is heat conduction; when the underside is hot side, the heat transfer mode is dependent on the Ra (Rayleigh) number (the product of $\mathrm{Gr}$ (Grashof) and $\operatorname{Pr}$ (Prandtl)) which is given by [55]

$$
\mathrm{Ra}=\frac{g \beta_{\text {air }} \Delta T L^{3}}{v_{\text {air }}^{2}} \frac{v_{\text {air }}}{a_{\text {air }}}=\frac{g \beta_{\text {air }} \Delta T L^{3}}{a_{\text {air }} v_{\text {air }}},
$$

where $\beta_{\text {air }} a_{\text {air }} v_{\text {air }}$ and $g$ are the coefficient of cubical expansion, thermal diffusivity and kinematic viscosity of air, and gravitational acceleration.
(1) When Ra $<1700$, the heat transfer is heat conduction and then the thermal resistance is

$$
R_{\mathrm{cdg}}=\frac{L}{A_{\mathrm{g}} k_{\mathrm{air}}}=\frac{L}{A_{\mathrm{cp}} k_{\mathrm{air}}(1-\theta)} .
$$

(2) When $\mathrm{Ra} \geq 1700$, the heat conduction develops to natural convection and then the thermal resistance is

$$
R_{\mathrm{cvg}}=\frac{1}{h_{\mathrm{cvg}} A_{\mathrm{g}}},
$$

where

$$
\begin{aligned}
& h_{\mathrm{cvg}}=\mathrm{Nu}_{\text {air }} / L, \\
& \mathrm{Nu}=0.59 \mathrm{Ra}^{0.4}, \quad 1.7 \times 10^{3} \leq \mathrm{Ra}<7.0 \times 10^{3}, \\
& \mathrm{Nu}=0.212 \mathrm{Ra}^{1 / 4}, \quad 7.0 \times 10^{3} \leq \mathrm{Ra}<3.2 \times 10^{5}, \\
& \mathrm{Nu}=0.061 \mathrm{Ra}^{1 / 3}, \quad \mathrm{Ra} \geq 3.2 \times 10^{5} .
\end{aligned}
$$

For this case, the Grashof number $\mathrm{Ra}<1700$, so the heat transfer through the air gap is heat conduction.

\section{References}

[1] A.F. Ioffe, Semiconductor, Thermoelements and Thermoelectric Cooling, Infosearch, London 1957.

[2] D.M. Rowe, CRC Handbook of Thermoelectrics, 1st ed., CRC Press, Boca Raton 1995.

[3] S.B. Riffat, X. Ma, Appl. Thermal Eng. 23, 913 (2003).

[4] J.P. Heremans, V. Jovovic, E.S. Toberer, A. Saramat, K. Kurosaki, A. Charoenphakdee, S. Yamanaka, G.J. Snyder, Science 321, 554 (2008).

[5] L.E. Bell, Science 321, 1457 (2008).

[6] K. Atik, Thermoeconomic Optimization in the Design of Thermoelectric Cooler, Karabuk, Turkiye 2009.

[7] S.W. Angrist, Direct Energy Conversion, 4th ed., Allyn and Bacon, Boston 1992.

[8] V. Radcenco, Generalized Thermodynamics, Editura Techica, Bucharest 1994.

[9] A. Bejan, Advanced Engineering Thermodynamics, 3rd ed., Wiley, Hoboken, N.J. 2006.

[10] F.L. Tan, S.C. Fok, Energy Convers. Manag. 49, 1715 (2008).

[11] M. Yamanashi, J. Appl. Phys. 80, 5494 (1996).

[12] J. Chen, Z. Yan, L. Wu, Energy, The Int. J. 22, 979 (1997).

[13] B.J. Huang, C.J. Chin, C.L. Duang, Int. J. Refriger. 23, 208 (2000).

[14] X.C. Xuan, Energy Convers. Manag. 44, 399 (2003).

[15] A. Bejan, Entropy Generation through Heat and Fluid Flow, Wiley, New York 1982.

[16] B. Andresen, Finite-Time Thermodynamics: Physics Laboratory II, University of Copenhagen, Copenhagen 1983.

[17] M. Feidt, Thermodynamique et Optimisation Energetique des Systems et Procedes, 2nd ed., Technique et Documentation, Lavoisier, Paris 1996.

[18] J.M. Gordon, K.C. Ng, Cool Thermodynamics, Cambridge Int. Science Publishers, Cambridge 2000. 
[19] A.M. Tsirlin, Irreversible, Estimates of Limiting Possibilities of Thermodynamic and Microeconomic Systems, Nauka, Moscow 2003.

[20] D.C. Agrawal, Eur. J. Phys. 30, 1173 (2009).

[21] D.C. Agrawal, Eur. J. Phys. 30, 587 (2009).

[22] D.C.M.V. Agrawal, Eur. J. Phys. 30, 295 (2009).

[23] M. Feidt, Entropy 11, 529 (2009).

[24] S. Sieniutycz, J. Jezowski, Energy Optimization in Process Systems, Elsevier, Oxford 2009.

[25] R. Ebrahimi, Acta Phys. Pol. A 117, 887 (2010).

[26] R. Ebrahimi, J. Am. Sci. 6, 113 (2010).

[27] B. Andresen, Angew. Chem. Int. Ed. 50, 2690 (2011).

[28] R. Ebrahimi, Math. Comp. Model 53, 1289 (2011).

[29] R. Ebrahimi, J. Energy Institute 84, 30 (2011).

[30] G.J. Vella, L.B. Harris, H.J. Goldsmid, Solar Energy 18, 355 (1976).

[31] S. Goktun, Energy Convers. Manag. 36, 1197 (1995).

[32] X.C. Xuan, K.C. Ng, C. Yap, H.T. Chua, Int. J. Heat Mass Tranfer 45, 5159 (2002).

[33] M. Huang, R. Yen, A. Wang, Int. J. Heat Mass Tranfer 48, 413 (2005).

[34] X.C. Xuan, Semicond. Sci. Tech. 17, 114 (2002).

[35] S.B. Riffat, G. Qiu, Appl. Thermal Eng. 24, 1979 (2004).

[36] J. Luo, L. Chen, F. Sun, C. Wu, Energy Convers. Manag. 44, 3197 (2003).

[37] L. Chen, J. Li, F. Sun, C. Wu, J. Appl. Phys. 98, 34507 (2005).

[38] Y. Cheng, W. Lin, Appl. Thermal Eng. 25, 2983 (2005).

[39] Y.G. Gurevich, I. Lashkevych, Int. J. Thermal Sci. 48, 2080 (2009).
[40] I. Lashkevych, C. Cortes, Y.G. Gurevich, J. Appl. Phys. 105, 53705 (2009).

[41] X. Chen, B. Lin, J. Chen, Appl. Energy 83, 681 (2006).

[42] N.M. Khattab, E.T.E. Shenawy, Energy Convers. Manag. 47, 407 (2006).

[43] L. Chen, F. Meng, F. Sun, J. Power Energy 223, 329 (2009).

[44] L. Chen, F. Meng, F. Sun, Rev. Mex. Fis. 55, 282 (2009).

[45] F. Meng, L. Chen, F. Sun, J. Energy Inst. 83, 108 (2010).

[46] F. Meng, L. Chen, F. Sun, Math. Comp. Model. 52, $586(2010)$.

[47] F. Meng, L. Chen, F. Sun, Indian J. Pure Appl. Phys. 48, 731 (2010).

[48] J.G. Vian, D. Astrain, Appl. Thermal Eng. 29, 3319 (2009).

[49] J.G. Vian, D. Astrain, Appl. Thermal Eng. 29, 1935 (2009).

[50] F. Incropera, D.D. Witt, Fundamentals, of Heat and Mass Transfer, 6th ed., Wiley, New York 2007.

[51] D.M. Rowe, G. Min, J. Power Sources 73, 193 (1998).

[52] Melcor, Thermoelectric Handbook, Available from: http://www.Laridtech.com , 2010.

[53] Ferrotec, http://www.Ferrotec.com.cn , 2010.

[54] A. Bejan, Convection Heat Transfer, 3th ed., Wiley, New York 2004.

[55] Y.A. Cengel, Heat Transfer - A Practical Approach, McGraw-Hill, New York 1998. 